\title{
Occurrence of Solar Radio Burst Fine Structures in 1-7.6 GHz Range Associated with CME Events
}

\author{
Yihua Yan, Yuying Liu, Zhijun Chen, Qijun Fu, Chengming Tan, \\ Shujuan Wang \& Jian Zhang $\dagger$
}

National Astronomical Obsevatories, Chinese Academy of Sciences, A20 Datun Road, Chaoyang District, Beijing 100012, China

email: yyh@bao.ac.cn

\begin{abstract}
The solar radio bursts and accompanying fine structures recorded by spectrometers at Huairou, Beijing during 1999-2003 are presented. The spectrometers are with high temporal $(5-10 \mathrm{~ms})$ and spectral $(4-20 \mathrm{MHz})$ resolutions. We found 91 radio burst events that occurred within half hour of the onset of the CME events which cause solar energetic particle events. The associations of radio fine structures with $\mathrm{CME}$ events are discussed.
\end{abstract}

Keywords. Sun: corona, Sun: coronal mass ejections (CMEs), Sun: Radio

\section{Introduction}

Radio observations at decimetric- and centimeter wavelengths provide important information for inferring fundamental processes of energy release, particle acceleration and particle transport in solar activities (Bastian et al. 1998). Temporal fine structures (FS) of solar radio emission in solar flares have been found in various wavebands for several decades (Allaart, et al. (1990), Bruggmann, et al. (1990), Benz, et al. (1992), Sawant, et al. (1994), Isliker \& Benz (1994), Jiricka, et al. (2001), Fu, et al. (2004a)). They are considered to be related to primary energy release processes, etc. (Bastian et al. 1998). The Solar Radio Broadband Dynamic Spectrometer (SRBS) of China is the first instrument in microwave to acquire dynamic spectrums of solar bursts with the combination of wide frequency coverage $(0.7-7.6 \mathrm{GHz})$, high temporal resolution, high spectral resolution, and high sensitivity (Fu et al. 2004b). We compare the occurrence of FSs in 1-7.6 $\mathrm{GHz}$ range with flare/CME events.

FSs in $>1 \mathrm{GHz}$ range are categoried by Isliker \& Benz (1994), Jiricka, et al. (2001), $\mathrm{Fu}$, et al. (2004a), etc., into different types. Kliem, et al. (2000) have found that the radio drifting pulsation structure are associated with CME initial process. Such structure are typical features for the active region NOAA 9077 during its passage over solar disk (Karlicky, et al. (2001), Wang, et al. (2001a)), and Wang, et al. (2001b) found that various radio FSs occurred during different phases for the Bastille Day event. The time sequence of the radio emission was analyzed by comparing with the hard X-rays (HXRs) and the soft X-rays (SXRs) in this flare. After the maxima of the X-rays, the radio emission in the range 1.0-7.6 GHz reached maxima first at the higher frequency, then drifted to the lower frequency. This comparison suggested that the flare included three successive processes: firstly the X-rays rose and reached maxima at 10:10-10:23 UT, accompanied by fine structures only in the lower altitude regime (range 2.6-7.6 GHz); secondly the

$\dagger$ Dept of Astronomy, Beijing University, Beijing, China 


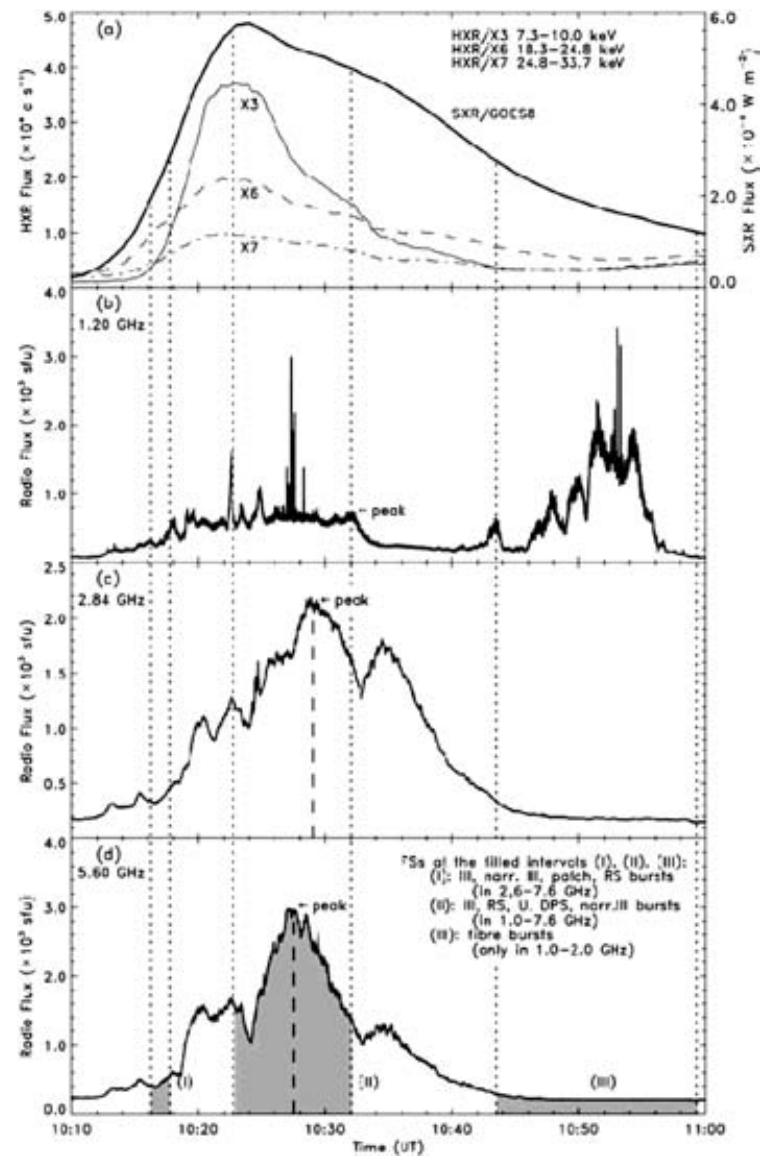

Figure 1. Time profiles of the HXRs and SXR and the radio emission at several typical frequencies during 10:00-11:00 UT on 14 July 2000. (a) HXRs by FY-2 satellite and GOES SXR; (b) $1.2 \mathrm{GHz}$; (c) $2.84 \mathrm{GHz}$; and (d) $5.60 \mathrm{GHz}$. The filled areas in (d) indicate three intervals only in which time interval many radio FSs occurred. During (I) 10:10-10:23 UT, accompanied by fine structures only in the range $2.6-7.6 \mathrm{GHz}$ (II) many fine structures over the range 1.0-7.6 $\mathrm{GHz}$ at 10:23-10:34 UT (III) a decimetric type IV burst and its associated FSs (fibers) in the range 1.0-2.0 GHz appeared after 10:40 UT (Wang et al. 2001b).

microwave radio emission reached maxima accompanied by many fine structures over the whole altitude (range 1.0-7.6 GHz) at 10:23-10:34 UT; then a decimetric type IV burst and its associated FSs (fibers) in the high altitude regime (range 1.0-2.0 GHz) appeared after 10:40 UT. For the Oct-Nov 2003 flare/CME events, various radio FSs were also found appearing at different phases of the flare/CME process (Tan, et al. (2004)).

The temporal relationship between CMEs and associated flares is of great importance to understanding the origin of CMEs. Zhang, et al. (2001) have studied this issue using SOHO/LASCO and EIT observations. The association of radio type II bursts and CMEs have been found for decades (Cane (1984), Aurass, (1992)), and Chernov \& Markeev (1997) discussed radio FSs in metric wave range associated with CMEs. Here the occurrence of FSs in 1-7.6 GHz with respect to the earth-effective CMEs, that cause solar energetic particle events, is statistically analyzed regardless the FS types. We first introduce the instruments and observations in $\S 2$. Then in $\S 3$ we present the statistical results and finally we draw our conclusions in $\S 4$. 
Table 1. The Solar Radio Spectrometers at Huairou/NAOC

\begin{tabular}{lccc}
\hline Frequency range: & $1.0-2.0 \mathrm{GHz}$ & $2.6-3.8 \mathrm{GHz}$ & $5.2-7.6 \mathrm{GHz}$ \\
\hline Temporal resolution: & $\begin{array}{c}5 \mathrm{~ms} \text { (after June 2002) } \\
\text { 20ms (before Dec. 2001) }\end{array}$ & $8 \mathrm{~ms}$ & $5 \mathrm{~ms}$ \\
\hline $\begin{array}{l}\text { Frequency resolution } \\
\text { (MHz/chan.): }\end{array}$ & $\begin{array}{c}4 / 240 \text { (after June 2002) } \\
20 / 50 \text { (before Dec. 2001) }\end{array}$ & $10 / 120$ & $20 / 120$ \\
\hline Sensitivity: & $3 \%$, & $2 \%$, & $2 \% \mathrm{~S}_{\text {quiet Sun }}$ \\
\hline Dynamic range: & $10 \mathrm{~dB}$ above 3 or $2 \% \mathrm{~S}_{\text {quiet Sun }}$ \\
\hline Polarizations: & \multicolumn{2}{c}{ LHCP, RHCP } \\
\hline Observing time: & $22-10 \mathrm{UT}$ (Summer), 0-8UT (Winter) \\
\hline
\end{tabular}

Table 2. Observed solar radio bursts during 1999-2003 at Huairou/NAOC

\begin{tabular}{lccc}
\hline Frequency range $(\mathrm{GHz}):$ & $1.0-2.0$ & $2.6-3.8$ & $5.2-7.6$ \\
\hline Number of Burst Events: & 729 & 1616 & 1198 \\
Number of Fine Structures: & 110 & 131 & 48 \\
\hline
\end{tabular}

\section{Instruments and Observations}

Since 1994, a broadband solar radio spectrometer had been developed in China, with a frequency coverage of $0.7-7.6 \mathrm{GHz}$, a frequency resolution of $1-10 \mathrm{MHz}$, and a temporal resolution of $1-10 \mathrm{~ms}(\mathrm{Fu}$, et al. $(2004 \mathrm{~b}))$. This instrument is composed of multibands spectrometers and the three spectrometers at 1.0-2.0 GHz, 2.6-3.8 GHz, and 5.27.6 GHz are located at Huairou Solar Observing Station of National Astronomical Observatories, Chinese Academy of Sciences (NAOC). The radio environment has been measured and calibration techniques are developed to ensure reliable observations (Yan, et al. (2002), Sych \& Yan (2002)).

The performance of the spectrometers is very powerful in detecting radio fine structures. Table 1 shows the description of the spectrometers at Huairou (Ji, et al. (2000), Ji, et al. (2003), Fu, et al. (2004b)).

Many events have been observed since the Chinese Solar Radio Broadband Spectrometers have been put into operation. The radio events observed at Huairou/NAOC during 1999 to 2003 is listed in Table 2.

The radio FSs have also been observed as shown in the above table. Figure 1 shows the occurrence of radio FSs associated with the flare/CME process for the Bastille Day event. For the Oct-Nov 2003 event we also observed FSs during flare/CME process (Tan, et al. (2004), Wang, et al. (2004)). Here we show the radio zebra pattern and spike fine structures during the rising phase of the 26 October 2003 event in Figure 2.

The CME list is obtained from SOHO/LASCO catalogue (http://cdaw.gsfc.nasa. gov/ CME_list/) and the LASCO instrument is introduced in detail by Brueckner, et al. (1995).

\section{Statistic Results}

As mentioned above, we mainly analyze associations of the occurrence of FSs in 17.6 GHz with respect to the earth-effective CMEs, i.e., that cause solar energetic particle 


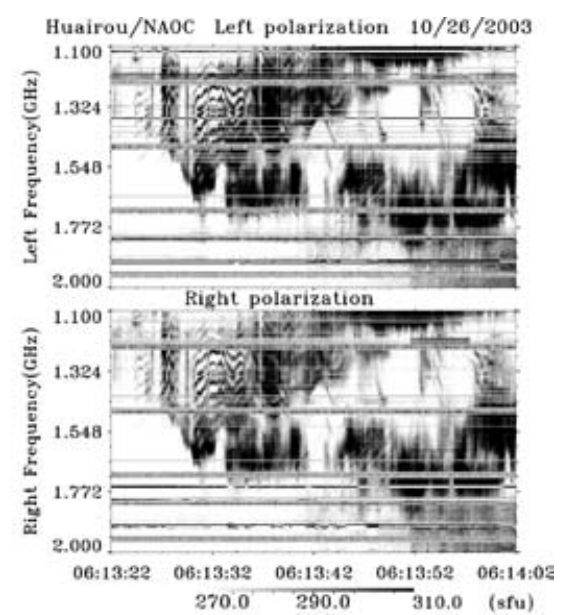

(a)

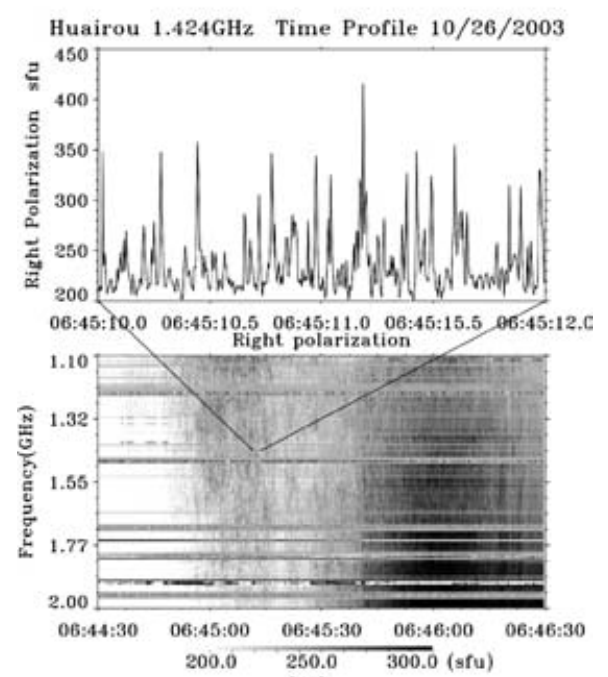

(b)

Figure 2. The radio (a) zebra pattern and (b) spike fine structures during the rising phase of the 26 October 2003 event.

events, regardless the FS types. Therefore we chose the radio burst events that occurred within half hour of the onset of the CME events.

During 1997-2003, there were 91 radio burst events in the 1-7.6 GHz range selected which are associated with the CME events, among which 86 were accompanying Type II/ IV bursts as shown in Table 3. All these 91 events associated with GOES X-ray flares as listed in Table 3 as well. Among 91 events, 60 (66\%) events have burst in all 3 bands employed by Huairou/NAOC spectrometers: 14/18 (78\%) for X-class flares 39/57 (68\%) for M-class flares and 7/16 (44\%) for C-class flares. 78 out of 91 events $(85.7 \%)$ were associated with $\mathrm{H} \alpha$ flares and the classification is shown in Table 4. If we look at the radio burst events and the GOES X-rays flares we can seen that most of them are radio complex events as shown in Table 5. The importance of the radio flux intensity at $2.84 \mathrm{GHz}$ for these events are listed in Table 6 . It can be seen that most are strong radio burst 
Table 3. Radio bursts (in 1-7.6 GHz) associated with CMEs and Type II/IV bursts and SXR flares

\begin{tabular}{c|cc|ccc}
\hline CMEs & Type II & Type IV & X & M & C \\
\hline 91 & 49 & 37 & 18 & 57 & 16 \\
$(100 \%)$ & $(54 \%)$ & $(41 \%)$ & $(20 \%)$ & $(62 \%)$ & $(18 \%)$ \\
\hline
\end{tabular}

Table 4. Radio bursts (in 1-7.6 GHz) associated with CMEs and $\mathrm{H} \alpha$ flares

\begin{tabular}{cccccccc}
\hline Total event & 3B & 2B & 1B & SF & $1 \mathrm{~N}$ & others & no flare \\
\hline 91 & 4 & 17 & 7 & 13 & 17 & 20 & 13 \\
\hline
\end{tabular}

Table 5. Classification of soft X-ray flares

\begin{tabular}{cccccccc}
\hline GOES flare & Event & $47 \mathrm{~GB}$ & $45 \mathrm{C}$ & $5 \mathrm{~S}$ & $3 \mathrm{~S}$ & $1 \mathrm{~S}$ & Other \\
\hline X class & 18 & 16 & 1 & 1 & 0 & 0 & 0 \\
M class & 57 & 15 & 27 & 4 & 9 & 1 & 1 \\
C class & 16 & 0 & 6 & 6 & 4 & 0 & 0 \\
\hline Total & 91 & 31 & 34 & 11 & 13 & 1 & 1 \\
\hline
\end{tabular}

events. This is agreeable to the selection that the CME events are all earth-directed and caused SEP events.

In all 91 events there are $38(41.7 \%)$ containing FSs (or FS groups). The 26 FSs occurred during rising phase, 16 during maximum, and 8 in decaying phase (there are overlaps for FS occurrence). Note here a FS event may contain different types of FSs as we mentioned above. The detailed description of these events are listed in Table 7 . We have also analyzed the time sequences of these flare/CME event and the results are listed in Table 8. It can be seen that most events are in radio burst-SXR flare maximum-CME time sequences. Please note that radio bursts and flares are measured at their peak time whereas the CME onsets are measured according to their $\mathrm{C} 2 / \mathrm{C} 3$ onset time. Therefore it deserves careful analysis of these time sequence relationship.

\section{Conclusions}

In summary, during 1997-2003, there were 91 radio burst events in the $1-7.6 \mathrm{GHz}$ range selected which are associated with the CME events that caused SEP events. The CME was simply chosen when they are half hour apart to the radio bursts and SXR flares.

(1) All the events are associated with soft X-ray flares (18 X class, $57 \mathrm{M}$ class and 16 C class events), 78 are associated with $\mathrm{H} \alpha$ flares, 49 associated with Type II burst and 37 with Type IV burst.

(2) In the 91 CME-associated radio burst events, 60 (66\%) have burst in all 3 frequency bands: 14/18 (78\%) for X-class 39/57 (68\%) for M-class and 7/16 (44\%) for C-class. Most of them are of complex radio burst profiles (65 events) and with strong intensity (71 events with $>100$ s.f.u. at $2.84 \mathrm{GHz}) .50$ events $(65 \%)$ were in a time sequence of "radio burst" $\rightarrow$ "SXR flare" $\rightarrow$ "CME onset".

(3) There are 38 (41.7\%) event containing FSs (or FS groups). Most radio FSs occurred during rising phase of flare/CMEs, and the occurrence of FSs decreases as frequency 
Table 6. Solar Radio Flux at $2.84 \mathrm{GHz}$ of the events

\begin{tabular}{cccccc}
\hline $\begin{array}{c}\text { Flux range } \\
\text { (s.f.u.) }\end{array}$ & $<100$ & $100-500$ & $500-1000$ & $1000-5000$ & $>5000$ \\
\hline Events & 20 & 40 & 14 & 16 & 1 \\
\hline
\end{tabular}

Table 7. Occurrence of radio FSs in different burst phases

\begin{tabular}{cccc}
\hline Phases: & rising & peak & decay \\
\hline $1-2 \mathrm{GHz}$ & 19 & 15 & 4 \\
$2.6-3.8 \mathrm{GHz}$ & 14 & 3 & 3 \\
$5.2-7.6 \mathrm{GHz}$ & 5 & 2 & 1 \\
\hline
\end{tabular}

Table 8. Time sequences of radio burst/CME/falres.

\begin{tabular}{lcc}
\hline Radio burst maximum $\rightarrow$ SXR flare maximum $\rightarrow$ CME & 59 & $(65 \%)$ \\
Radio burst maximum $\rightarrow$ CME $\rightarrow$ SXR flare maximum & 8 & $(9 \%)$ \\
SXR flare maximum $\rightarrow$ Radio burst maximum $\rightarrow$ CME & 12 & $(13 \%)$ \\
SXR flare maximum $\rightarrow$ CME $\rightarrow$ Radio burst maximum & 2 & $(2 \%)$ \\
CME $\rightarrow$ Radio burst maximum $\rightarrow$ SXR flare maximum & 7 & $(8 \%)$ \\
CME $\rightarrow$ SXR flare maximum $\rightarrow$ Radio burst maximum & 3 & $(3 \%)$ \\
\hline
\end{tabular}

increases (19/14/5 FS events in rising, 15/3/2 at peak, and 4/3/1 when decay for $1-2$ $\mathrm{G} / 2.6-3.8 \mathrm{G} / 5.2-7.6 \mathrm{GHz}$ regime).

(4) If we consider a standard flare/CME model the above statistic results are consistent with such a scenario and radio fine structures may manifest initial phase signature, which is important to diagnose coronal parameters.

\section{Acknowledgements}

The work is supported by CAS, NSFC grants 10225313, 10333030, and MOST grant G2000078403. We acknowledge SOHO/LASCO for providing CME catalog used in this study.

\section{References}

Allaart, M. A. F., van Nieuwkoop, J., Slottje, C., \& Sondaar, L. H. 1990, Sol. Phys. 130, 183 Aurass, H. 1992, Ann. Geophys. 10, 359

Bastian, T. S., Benz, A. O., \& Gary, D. E. 1998, ARAA 36, 131

Benz, A. O., Su, H., Magun, A., \& Stehling, W. 1992, A\&A 93, 539

Brueckner, G. E., Howard, R. A., Koomen, M. J., \& et al. 1995, Sol. Phys. 162, 357

Bruggmann, G., Magun, A., Benz, A. O., \& Stehling, W. 1990, A\&ASS 240, 506

Cane, H. V. 1984, A\&A 140, 205

Chernov, G. P., \& Markeev, A. K. 1997, IAU JD 19, 16

Fu, Q., Yan, Y., Liu, Y., \& et al. 2004a, Chin. J. Astron. Astrophys. 4, 176

Fu, Q., Ji, H., Qin, Z., \& et al. 2004b, Sol. Phys. 222, 167

Isliker, II., \& Benz, A. 1994, A\&A 105, 205

Ji, II., Fu, Q., Liu, Y., \& et al. 2000, ACTA Astrophysica Sinica 20, 209

Ji, H., Fu, Q., Liu, Y., \& et al. 2003, Sol. Phys. 213, 359

Jiricka, T., \& et al. 2001, A\&\&A 375, 243

Karlicky, M., Yan, Y. H., Fu, Q. J., \& et al. 2001, A\&A 369, 1104

Kliem, B., Karlicky, M., \& Benz, A. O. 2000, A\&A 360, 715

Sawant, H. S., Fernandes, F. C. R., \& Neri, J. A. C. F. 1994, ApJS 90, 689 
Sych, R. A., \& Yan, Y. H. 2002, Chin. J. Astron. Astrophys. 2, 183

Tan, C.-M., Fu, Q.-j., Yan, Y.-H., \& Liu, Y-Y. 2004, Chin. J. Astron. Astrophys. 4, 205

Wang, S. J., Yan, Y. H., \& Fu, Q. J. 2001a, A\&A 370, L13

Wang, S. J., Yan, Y. II., Zhao, R. Z., \& et al. 2001b, Sol. Phys. 204, 153

Wang, S. J., \& et al. 2004, IAU Symp 226 (these proceedings)

Yan, Y., Tan, C., Xu, L., Ji, H., Fu, Q., \& Song, G. 2002, Since in China (Series A) 45, 89

Zhang, J., Dere, K. P., Howard, R. A., Kundu, M. R., \& White, S. M. 2001, ApJ 559, 452 